\title{
Experimental evidence of space charge driven resonances in high intensity linear accelerators
}

\author{
Dong-O Jeon* \\ Rare Isotope Science Project, Institute for Basic Science, Daejeon 34047, Republic of Korea
}

(Received 12 June 2015; published 12 January 2016)

\begin{abstract}
In the construction of high intensity accelerators, it is the utmost goal to minimize the beam loss by avoiding or minimizing contributions of various halo formation mechanisms. As a halo formation mechanism, space charge driven resonances are well known for circular accelerators. However, the recent finding showed that even in linear accelerators the space charge potential can excite the $4 \sigma=360^{\circ}$ fourth order resonance [D. Jeon et al., Phys. Rev. ST Accel. Beams 12, 054204 (2009)]. This study increased the interests in space charge driven resonances of linear accelerators. Experimental studies of the space charge driven resonances of high intensity linear accelerators are rare as opposed to the multitude of simulation studies. This paper presents an experimental evidence of the space charge driven $4 \sigma=360^{\circ}$ resonance and the $2 \sigma_{x(y)}-2 \sigma_{z}=0$ resonance of a high intensity linear accelerator through beam profile measurements from multiple wire-scanners. Measured beam profiles agree well with the characteristics of the space charge driven $4 \sigma=360^{\circ}$ resonance and the $2 \sigma_{x(y)}-2 \sigma_{z}=0$ resonance that are predicted by the simulation.
\end{abstract}

DOI: 10.1103/PhysRevAccelBeams.19.010101

\section{INTRODUCTION}

Recently many high intensity linear accelerators have been constructed or are under construction such as the SNS (USA) [1], the J-PARC (Japan) [2], the KOMAC (Korea) [3], the CSNS (China) [4], the RISP (Korea) [5], and the FRIB (USA) [6]. For high intensity linear accelerators, a primary concern is potential damage and radio activation of accelerator components resulting from uncontrolled beam losses. A major source of loss is a beam halo that intercepts the bore of the linac and it is the utmost goal to minimize the beam loss of halo particles by avoiding or minimizing contributions of various halo formation mechanisms. Space charge driven resonances are of interest as halo formation mechanisms. Recently a few space charge driven resonances were studied for linear accelerators.

In the past, a lot of work has been done related to the envelope equation [7], the particle-core model [8-11] and the envelope instability $[12,13]$. The space charge driven $2 \nu_{x}-2 \nu_{y}=0$ coupling resonance study [14] between the two transverse planes in the circular accelerator reported in 1999 drew attention to the space charge driven resonances of high intensity accelerators, and the study was extended to high intensity linear accelerators such as [15]. The interest in space charge driven resonances of linear

\footnotetext{
*Corresponding author. jeond@ibs.re.kr

Published by the American Physical Society under the terms of the Creative Commons Attribution 3.0 License. Further distribution of this work must maintain attribution to the author $(s)$ and the published article's title, journal citation, and DOI.
}

accelerators was increased by the first finding reported in 2009 that the space charge potential can excite the $4 \sigma=$ $360^{\circ}$ fourth order resonance in high intensity linear accelerators [16] just like in circular accelerators, and that the $4 \sigma=360^{\circ}$ resonance emerges first rather than the better known envelope instability for initially well-matched beams. This resonance is excited for $90^{\circ}-\Delta \sigma \leq \sigma \leq$ $90^{\circ}$. Here $\sigma$ is the depressed phase advance per cell of the linac lattice and $\Delta \sigma$ is the stopband width of the fourth order resonance which depends on the space charge tune depression. This resonance was verified through the emittance measurement and reported in 2009 [17]. Recently, it was first found in 2015 that the space charge driven $6 \sigma=$ $720^{\circ}$ sixth order resonance is induced in high intensity linear accelerators when $\sigma$ is close to and below $120^{\circ}$ [18].

In the design of high intensity linear accelerators, designers have followed the rule to keep $\sigma_{o}<90^{\circ}$ in fear of the envelope instability [19]. For example the SNS designers followed this rule (see Fig. 10 in [1]). However the studies of the $4 \sigma=360^{\circ}$ fourth order resonance $[16,17]$ indicate that designers should keep $\sigma_{o}<90^{\circ}$ because of the $4 \sigma=360^{\circ}$ resonance as well that is excited even for wellmatched beams. Moreover, some recent linac designs such as [5,6] adopted $\sigma_{o}<\sim 120^{\circ}$ for the upstream sections of the linac because $\sigma_{o}$ decreases very quickly from $\sim 120^{\circ}$ to below $90^{\circ}$ as the beam is accelerated, and the associated resonance effect is negligible. An in-depth study of the space charge $4 \sigma=360^{\circ}$ fourth order resonance and the envelope instability was recently reported [20].

In this paper, the experimental results are reported for the space charge driven $4 \sigma=360^{\circ}$ resonance and $2 \sigma_{x(y)}-$ $2 \sigma_{z}=0$ resonance. The experiments are based on multiple 
wire-scanner profile measurements using the Spallation Neutron Source (SNS) Coupled Cavity Linac (CCL). The main difficulties of the experiment are to generate a beam with little halo and to maintain a nice beam quality into the CCL. Otherwise, the induced halos by other mechanisms can overshadow the effects of the resonances of interest. In Sec. II, we present the experimental evidence of the space charge driven $4 \sigma=360^{\circ}$ resonance. In particular, the experimental data show the tendency that the spatial extent of beam halo decreases as $\sigma$ gets very close to $90^{\circ}$, just as predicted by the simulation. In subsections II B and III B, it is described how a beam with little halo into the CCL is generated and how a nice beam quality is maintained by matching well between the sections of the linear accelerator. In Sec. III, we present the experimental evidence of the space charge $2 \sigma_{x(y)}-2 \sigma_{z}=0$ coupling resonance. In Sec. IV, follows the conclusion.
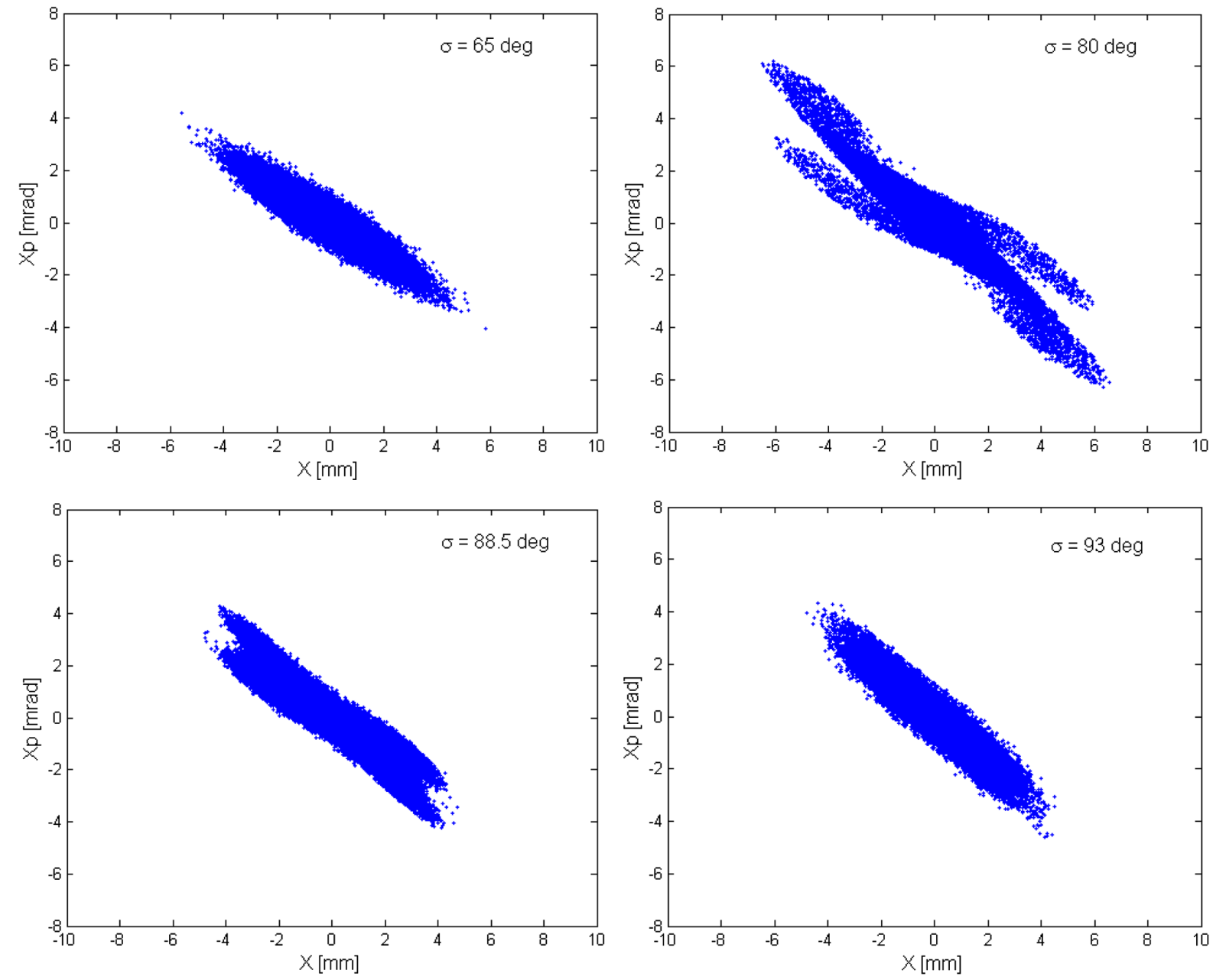

FIG. 1. Plots of output beam distributions for four different values of $\sigma$, depressed phase advance per cell of the CCL lattice. The top left plot displays the beam distribution for the lattice with depressed phase advance per cell $\sigma=65^{\circ}\left(\sigma_{\mathrm{O}}=85^{\circ}\right)$, the top right plot for $\sigma=80^{\circ}\left(\sigma_{\mathrm{O}}=100^{\circ}\right)$, the bottom left plot for $\sigma=88.5^{\circ}\left(\sigma_{\mathrm{O}}=108.5^{\circ}\right)$, the bottom right plot for $\sigma=93^{\circ}\left(\sigma_{\mathrm{O}}=113^{\circ}\right)$. The difference between the depressed and undepressed phase advance is $20^{\circ}$. The beam distribution change should be noted as the phase advance changes from $\sigma=80^{\circ}$ to $\sigma=88.5^{\circ}$, which presents a unique feature measurable with wire-scanners. Quadrupole gradients are adjusted to maintain pretty constant phase advance $\sigma$ over the CCL. 
the entire CCL for a given $\sigma$ value. The gradients of CCL quadrupoles thus obtained are used to set up the actual linac for $\sigma=65^{\circ}, 80^{\circ}, 88.5^{\circ}$. However the $\sigma=93^{\circ}$ lattice is not used due to the interlock of the quadrupole power supplies, because the required current surpassed the interlocked values.

The numerical simulation is performed using the PARMILA code [21] with 50000 macro-particle Gaussian beam distribution with normalized rms emittance $\varepsilon_{x}=\varepsilon_{y}=$ $0.301 \mathrm{~mm} \mathrm{mrad}, \varepsilon_{z}=0.394 \mathrm{~mm} \mathrm{mrad}$. These input emittance values are obtained from the measured transverse and longitudinal beam profile data from wire-scanners and bunch shape monitors. For this beam, it is computed that $\sigma_{\mathrm{o}}-\sigma=20^{\circ}$ numerically using the PARMILA code. It should be noted that the value of $\sigma_{\mathrm{O}}-\sigma$ depends on the beam current. The phase advance is computed numerically from the tracked beam distributions.

Simulations show that this resonance is pretty strong and develops fast for the 38-mA beam. For instance, this resonance induces an rms emittance growth of $67 \%$ for the $\sigma=80^{\circ}\left(\sigma_{\mathrm{O}}=100^{\circ}\right)$ lattice according to the multiparticle simulation. Simulations show that distinct resonance features can develop in the beam profiles depending on the phase advance per cell $\sigma$. Figure 1 shows the beam distributions at the location of a wire-scanner in the downstream CCL for a few $\sigma$ values used for the experiment.

The top left plot shows the output beam distribution for the $\sigma=65^{\circ}\left(\sigma_{\mathrm{O}}=85^{\circ}\right)$ lattice, which is well below the resonance and does not show any sign of resonance effects. The top right plot is for the $\sigma=80^{\circ}\left(\sigma_{\mathrm{O}}=100^{\circ}\right)$ lattice, which shows well-developed resonance structures with particles trapped in the four resonance islands. The bottom left plot for the $\sigma=88.5^{\circ}\left(\sigma_{\mathrm{O}}=108.5^{\circ}\right)$ lattice, which shows that resonance islands shrink and move toward the origin compared with the $\sigma=80^{\circ}$ lattice case. The bottom right plot for the $\sigma=93^{\circ}\left(\sigma_{\mathrm{O}}=113^{\circ}\right)$ lattice, which is above the resonance and does not show any resonance effects again.

In particular, the comparison between the $\sigma=80^{\circ}$ and $\sigma=88.5^{\circ}$ cases shows an interesting feature of the $4 \sigma=360^{\circ}$ resonance. As the phase advance switches from $\sigma=80^{\circ}$ (the top right plot in Fig. 1) to $\sigma=88.5^{\circ}$ (the bottom left plot in Fig. 1), the corresponding change in the beam distribution leads to a distinct changes in the beam profiles which can be measured with wire-scanners: the intensity of beam shoulders increases while their spatial extent decreases (see the arrows in the top plot of Fig. 5). This is a unique feature that can be measured associated with the $4 \sigma=360^{\circ}$ resonance and is due to the fact that the resonance islands shrink and move toward the origin as $\sigma$ gets very close to $90^{\circ}$.

\section{B. Beam matching and incoming beam quality}

Figure 2 shows the schematic plot of the CCL and where the wire-scanners are installed. In the experiment, the beam matching is very crucial to minimize the possibility of beam mismatch and its associated halo formation. For each lattice with a given phase advance $\sigma$ value, the upstream four quadrupoles are adjusted to match the beam into the CCL by using the four wire-scanners installed in series for matching (WS104, 106, 108, 110 in Fig. 2). This matching procedure is described in detail in [22]. Figure 3 shows the results of beam matching to the CCL in the experiment for the three lattices with $\sigma=65^{\circ}, 80^{\circ}$, and $88.5^{\circ}$ respectively. Figure 3 shows that the beam is matched well into the CCL. The blue (red) solid dots represent the measured X (Y) beam sizes obtained from the five wire-scanners (WS104, 106, 108, 110, 204). Solid lines represent the TRACE3D

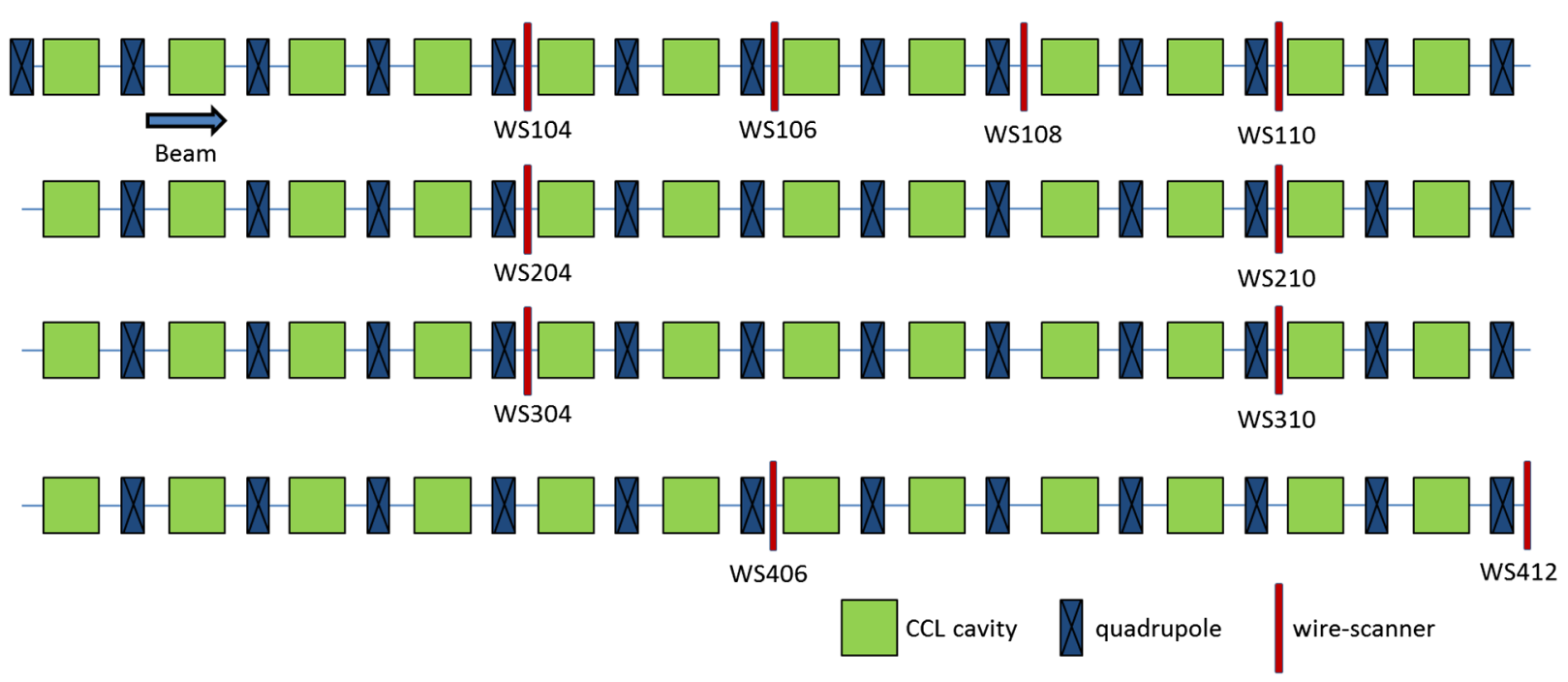

FIG. 2. Schematic plot of the CCL (Coupled Cavity Linac) and the wire-scanners. The top row represents the CCL module 1 and the beam travels from the left to the right and top to bottom. The CCL consists of four modules and ten wire-scanners are in use in total. The upstream four quadrupoles are used for the beam matching between the DTL (Drift Tube Linac) and the CCL. 

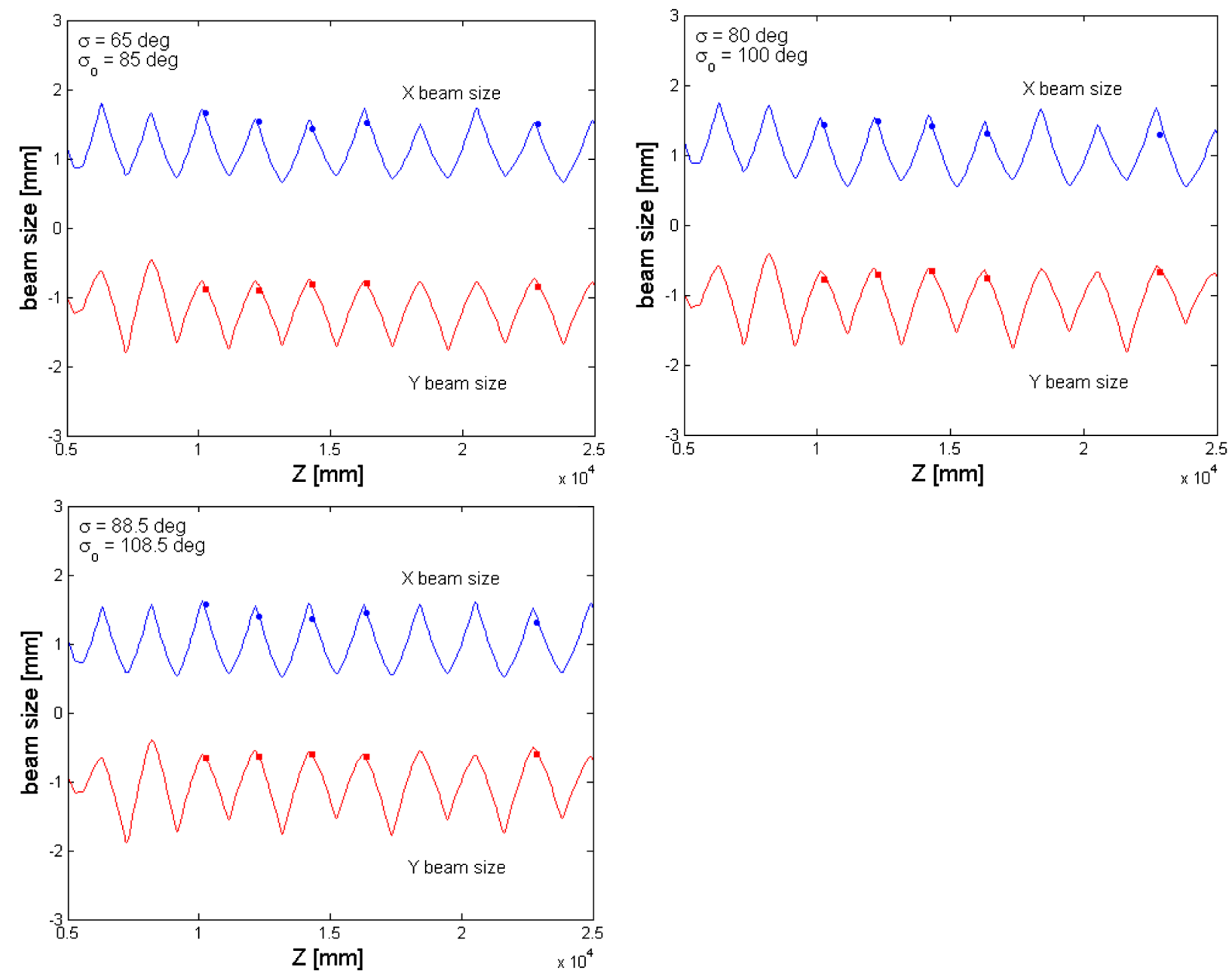

FIG. 3. Plots of beam matching to the CCL in the experiment for three phase advances. The beam is going from the left to the right. The top left plot is for the $\sigma=65^{\circ}\left(\sigma_{\mathrm{O}}=85^{\circ}\right)$ lattice, the top right plot for the $\sigma=80^{\circ}\left(\sigma_{\mathrm{O}}=100^{\circ}\right)$ lattice, the bottom left plot for the $\sigma=88.5^{\circ}\left(\sigma_{\mathrm{O}}=108.5^{\circ}\right)$ lattice. In the plots, $\sigma\left(\sigma_{o}\right)$ is the depressed (undepressed) phase advance per cell. The blue (red) solid dots are the measured X (Y) beam sizes obtained from the wire-scanners. Solid lines are the TRACE3D [23] fit to the wire-scanner beam size data, determining the beam parameters $(\varepsilon, \beta, \alpha)$ of the incoming beam. The upstream four wire-scanners in series from the left (WS104, 106, 108, 110 in Fig. 2) are used to do the beam matching into the CCL experimentally. It should be noted that the beam is matched well into the CCL for all the three cases. The beam current is $38 \mathrm{~mA}$ and the beam travels from the left to right.

code [23] fit to the beam size data obtained from wire-scanners for the 38-mA beam, which determines the incoming beam parameters $(\varepsilon, \beta, \alpha)$. The upstream four wire-scanners in series are used for the beam matching (the beam travels from the left to the right).

We suppress the beam tails and improve the quality of the beam going into the CCL by adopting the "round beam MEBT optics" in the MEBT (Medium Energy Beam Transport) which suppresses the halo formation induced by the optics of the SNS MEBT [24,25]. Both the simulation [24] and the experiment [25] show that the "round beam MEBT optics" reduces the X rms emittance and beam halo significantly. The quality of the beam going into the CCL can be measured with the four upstream CCL wire-scanners installed in series (WS104, 106, 108, 110 in Fig. 2). As shown in the measured profiles of Fig. 4, the beam going into the CCL has very little beam shoulders and has a pretty Gaussian-like density profile for all the wirescanners. The phase advance between the neighboring wire-scanners is $65^{\circ}$ (lattice with $\sigma=65^{\circ}$ ), which ensures detection of existing beam shoulders using the four wiresacnners in series. And Fig. 3 shows that the beam is matched well into the CCL for all the three cases with the $\sigma=65^{\circ}, 80^{\circ}$, and $88.5^{\circ}$. These two factors removes the possibility of halo formation due to a beam mismatch and existing beam tails of the beam going into the CCL.

\section{Experiment of space charge driven $4 \sigma=360^{\circ}$ resonance}

Once the beam matching is done for each CCL lattice with the $\sigma=65^{\circ}, 80^{\circ}$, and $88.5^{\circ}$ respectively, beam profiles are measured with all the wire-scanners in the CCL. It 

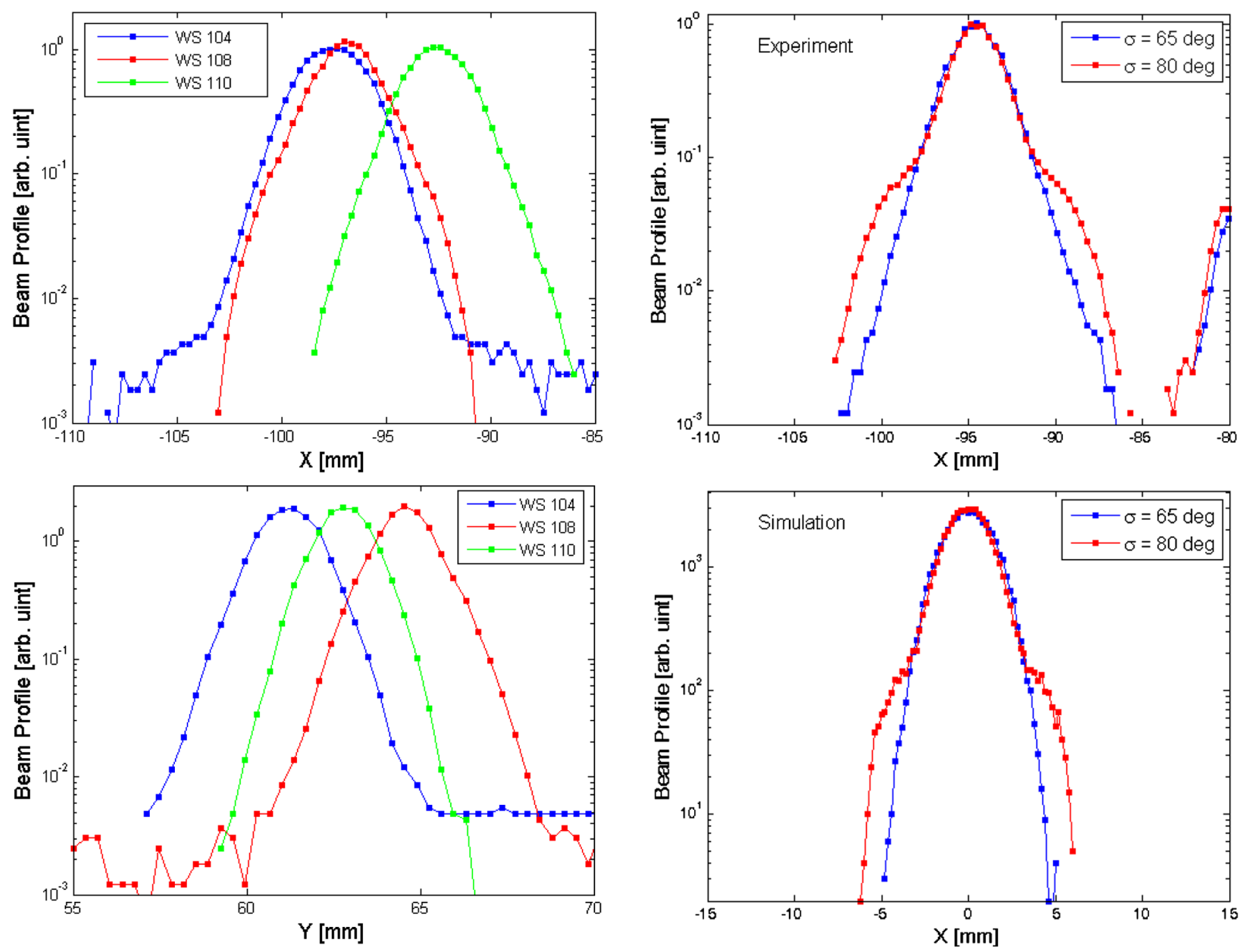

FIG. 4. Plots of measured beam profiles of the beam going into the CCL using the upstream CCL wire-scanners in series for the $\sigma=65^{\circ}\left(\sigma_{\mathrm{O}}=85^{\circ}\right)$ lattice. The top plot shows the $\mathrm{X}$ beam profiles in $\log$ scale obtained from the three wire-scanners, "WS104", "WS108", and "WS110" and the bottom plot shows the Y beam profiles from the same wire-scanners. These plots show the quality of the beam going into the CCL. Measured beam profiles indicate that the beam going into the CCL has very little beam shoulders. For the $\sigma=65^{\circ}$ lattice, there is no resonance effect coming into play in the CCL. These four wire-scanners installed in series are used for matching the beam into the CCL. The data of "WS106" were not saved properly to the file due to software problems during the experiment.

should be pointed out that there is no beam loss detected to the beam current monitors nor to the beam loss monitors for all the settings.

The measurements clearly show that significant beam shoulders (halo) are induced for the $\sigma=80^{\circ}$ lattice, not for the $\sigma=65^{\circ}$ lattice under the conditions: (i) the beam going into the CCL has initially little shoulders (see Fig. 3) and (ii) the beam is well matched into the CCL (see Fig. 2). Figure 5 shows the beam profiles for the experiment (the top

FIG. 5. Plots of the beam profiles for the $\sigma=65^{\circ}\left(\sigma_{\mathrm{O}}=85^{\circ}\right)$ and $\sigma=80^{\circ}\left(\sigma_{\mathrm{O}}=100^{\circ}\right)$ lattices in log scale. The top plot shows the beam profiles obtained from the wire-scanner measurement and the bottom plot from the simulation at the wire-scanner "WS304" location. As the simulation predicts in the bottom plot, one observes no beam shoulders for the $\sigma=65^{\circ}$ lattice in the blue curve in the experiment. On the other hand, one observes significant beam shoulders in the red curve for the $\sigma=80^{\circ}$ lattice. It should be noted that the beam core width from the measurement agrees well with the simulation. However the total beam width from the measurement is wider than that from the simulation. This is due to a lack of our knowledge of the input beam density distribution. Note that the measured "tail" in $-85 \mathrm{~mm} \sim-80 \mathrm{~mm}$ is due to the cross talking of $\mathrm{x}$ and $\mathrm{y}$ wires of wire-scanners.

plot) and the simulation (the bottom plot) in log scale for the $\sigma=65^{\circ}\left(\sigma_{\mathrm{O}}=85^{\circ}\right)$ and $\sigma=80^{\circ}\left(\sigma_{\mathrm{O}}=100^{\circ}\right)$ lattices at the downstream wire-scanner called "WS304". The top plot shows the beam profiles obtained from the experiment, and the bottom plot shows those from the simulation under the same conditions. The striking similarity should be noted between the experiment and simulation. In the experiment, 

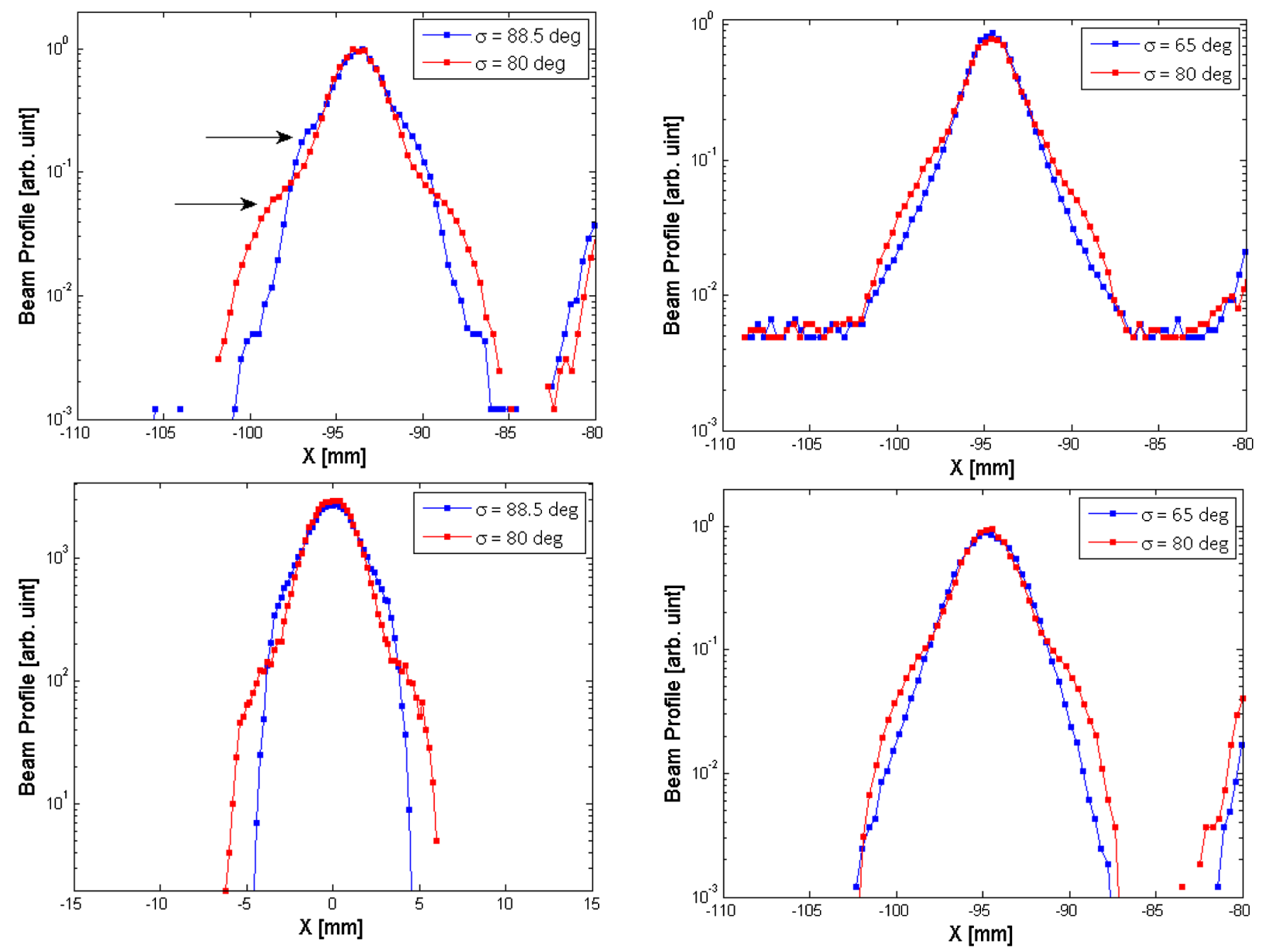

FIG. 6. Plots of the beam profiles for the $\sigma=88.5^{\circ}$ $\left(\sigma_{\mathrm{O}}=108.5^{\circ}\right)$ and $\sigma=80^{\circ}\left(\sigma_{\mathrm{O}}=100^{\circ}\right)$ lattices in log scale. The top plot shows the beam profiles obtained from the experiment and the bottom plot those from the simulation at the wirescanner "WS304". The agreement between the experiment and the simulation is remarkable. As predicted in the simulation, one observes in the experiment that for the $\sigma=88.5^{\circ}$ case the intensity of the beam shoulders increases while their spatial extent shrinks, compared with the $\sigma=80^{\circ}$ case (marked by the arrows). This is a pretty unique characteristic of the $4 \sigma=360^{\circ}$ space charge resonance. It should be noted that the beam core width from the measurement agrees well with the simulation. However the total beam width from the measurement is wider than that from the simulation. This is due to a lack of our knowledge of the input beam density distribution. Note that the measured "tail" in $-85 \mathrm{~mm} \sim-80 \mathrm{~mm}$ is due to the cross talking of $\mathrm{x}$ and $\mathrm{y}$ wires of wire-scanners.

one observes practically no beam shoulders for the $\sigma=65^{\circ}$ lattice, which is consistent with the numerical simulation. This is a proof that there is no resonance effect and no halo formation for the $\sigma=65^{\circ}$ lattice.

On the other hand, one observes strong growth of beam shoulders indicating substantial halo formation for the

FIG. 7. Plots of the measured beam profiles in log scale for the $\sigma=65^{\circ}\left(\sigma_{\mathrm{O}}=85^{\circ}\right)$ and $\sigma=80^{\circ}\left(\sigma_{\mathrm{O}}=100^{\circ}\right)$ lattices at two further downstream CCL wire-scanners. The top plot shows the beam profiles obtained from the wire-scanner "WS310" and the bottom plot from the wire-scanner "WS406". One observes formation of beam shoulders for the $\sigma=80^{\circ}$ lattice, while one observes no beam shoulders for $\sigma=65^{\circ}$ lattice. One observes the characteristics in the top plot of Fig. 5 of the $4 \sigma=360^{\circ}$ space charge resonance consistently at these wire-scanners "WS310" and "WS406". Note that the measured "tail" in $-85 \sim-80 \mathrm{~mm}$ is due to the cross talking of $\mathrm{x}$ and $\mathrm{y}$ wires of wire-scanners.

$\sigma=80^{\circ}$ lattice in the experiment and simulation, even though the beam is well matched into the CCL (see Fig. 2) and has little initial beam shoulders (see Fig. 3). This pronounced beam shoulder for the $\sigma=80^{\circ}$ lattice is caused by the $4 \sigma=360^{\circ}$ resonance. Simulations show that the resonance four-fold structures are already well developed at the wire-scanner "WS304" location (see the top left plot for $\sigma=80^{\circ}$ of Fig. 1). One should note that the measured core beam width agrees well with the simulation. However, the total beam width measured in the experiment is wider than that of the simulation. This difference is due to a lack of our 

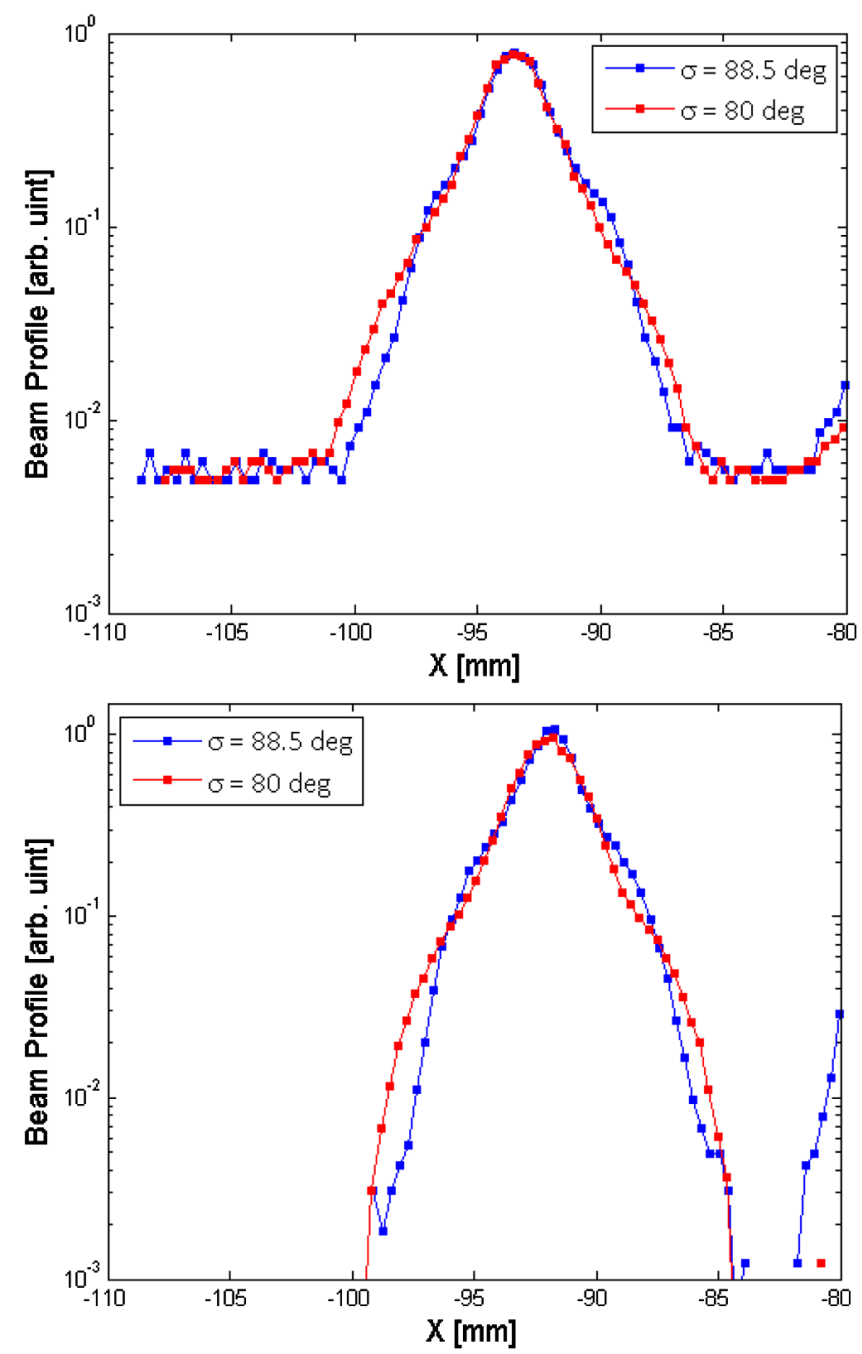

FIG. 8. Plots of the measured beam profiles in log scale for the $\sigma=88.5^{\circ}\left(\sigma_{\mathrm{O}}=108.5^{\circ}\right)$ and $\sigma=80^{\circ}\left(\sigma_{\mathrm{O}}=100^{\circ}\right)$ lattices at two further downstream CCL wire-scanners. The top plot shows the beam profiles obtained from the wire-scanner called "WS310" and the bottom plot from the wire-scanner "WS406". One observes that the intensity of the beam shoulders increases while their spatial extent decreases for the $\sigma=88.5^{\circ}$ case, compared with the $\sigma=80^{\circ}$ case. One observes the characteristics in the top plot of Fig. 6 of the $4 \sigma=360^{\circ}$ space charge resonance also at these downstream wire-scanners. Note that the measured "tail" in $-85 \sim-80 \mathrm{~mm}$ is due to the cross talking of $\mathrm{x}$ and $\mathrm{y}$ wires of wire-scanners.

knowledge of the input beam density distribution for we use a generated beam distribution of Gaussian density profile truncated at three standard deviations. Apparently the actual beam seems to have wider beam extent for the beam halo.

Now a comparison of the beam profiles for the $\sigma=80^{\circ}$ $\left(\sigma_{\mathrm{O}}=100^{\circ}\right)$ and $\sigma=88.5^{\circ}\left(\sigma_{\mathrm{O}}=108.5^{\circ}\right)$ lattices presents another evidence. Figure 6 displays the beam profiles in log scale for the $\sigma=80^{\circ}$ and $\sigma=88.5^{\circ}$ lattices at the wirescanner "WS304", showing the striking resemblance between the experiment (the top plot) and the simulation (the bottom plot). One needs to compare the beam

distribution for the $\sigma=80^{\circ}$ case (the top right plot of Fig. 1) and that for the $\sigma=88.5^{\circ}$ case (the bottom left plot of Fig. 1). As the resonance islands shrink and move toward the origin for the $\sigma=88.5^{\circ}$ case compared with the $\sigma=80^{\circ}$ case, subsequent changes in beam profiles take place: the intensity of the beam shoulders increases for the $\sigma=88.5^{\circ}$ case while their spatial extent decreases (see the arrows in Fig. 6), compared with the $\sigma=80^{\circ}$ case.
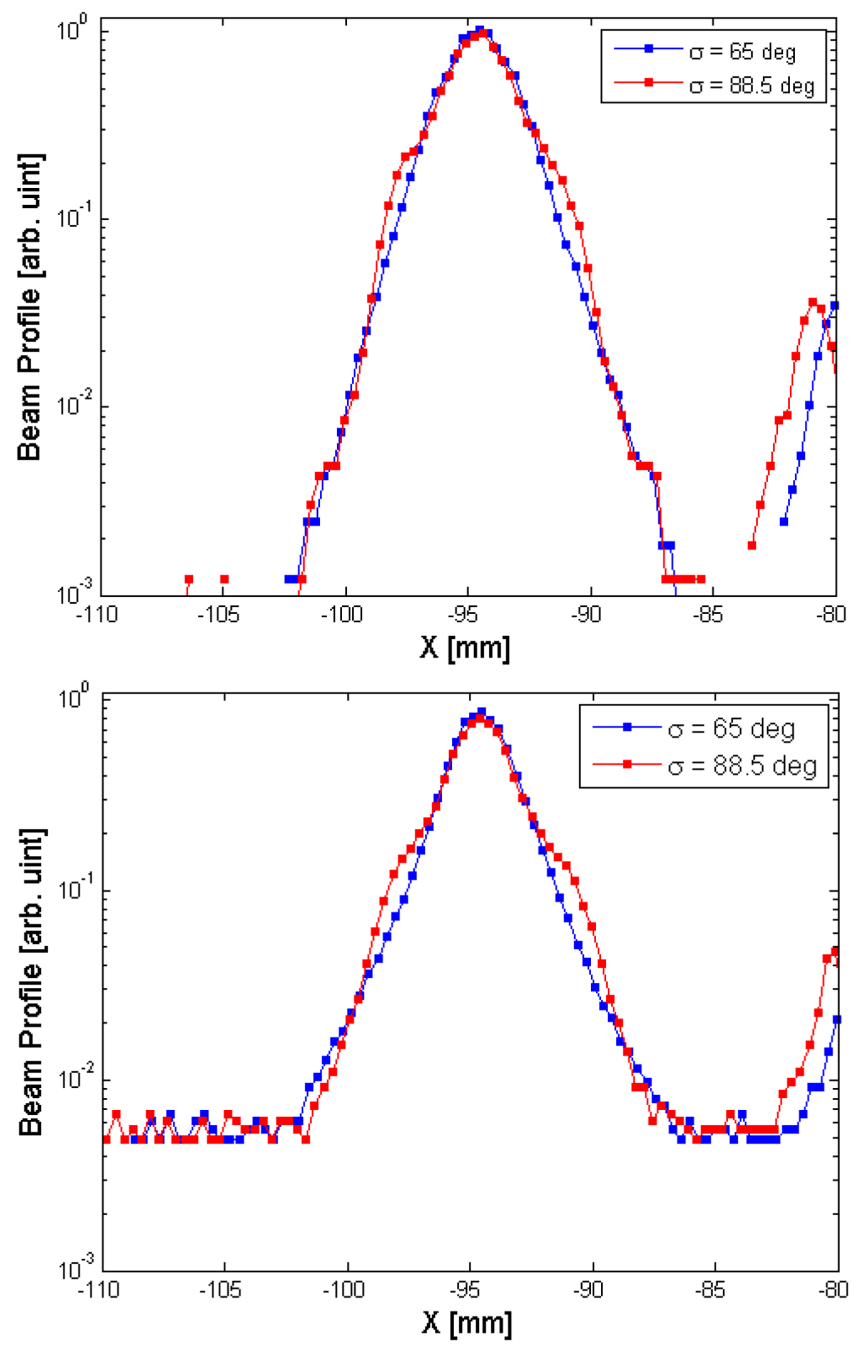

FIG. 9. Plots of the beam profiles for the $\sigma=65^{\circ}\left(\sigma_{\mathrm{O}}=85^{\circ}\right)$ and $\sigma=88.5^{\circ}\left(\sigma_{\mathrm{O}}=108.5^{\circ}\right)$ lattices in log scale obtained from the wire-scanners "WS304" (the top plot) and "WS310" (the bottom plot). These two wire-scanners are optically three cells apart. In case of the $\sigma=88.5^{\circ}$ lattice, they are $265.5^{\circ}$ apart. These plots show that the overall beam profile of the $\sigma=88.5^{\circ}$ lattice practically overlaps with that of $\sigma=65^{\circ}$ lattice except for the small beam shoulders generated by the beam particles populating the resonance islands. The plots of the $\sigma=88.5^{\circ}$ lattice shows that the spatial extent of beam halo decreases as $\sigma$ gets close to $90^{\circ}$. However the $\sigma=93^{\circ}$ lattice is not used due to the interlock of the quadrupole power supplies, because the required current surpassed the interlocked values. Note that the measured "tail" in $-85 \sim-80 \mathrm{~mm}$ is due to the cross talking of $\mathrm{x}$ and $\mathrm{y}$ wires of wire-scanners. 
Figures 5 and 6 manifest the consistent resonance characteristics both in the experiment and the simulation.

Measurements at the rest downstream wire-scanners also indicate that the same features persist that are observed at the wire-scanner "WS304" in Figs. 5 and 6. One observes consistent behavior of the resonance in all the downstream wire-scanner data: "WS304", "WS310" and "WS406". Figure 7 shows the comparison of beam profiles at "WS310" and "WS406" in log scale for the $\sigma=65^{\circ}$ and $\sigma=80^{\circ}$ lattices and Fig. 8 for the $\sigma=80^{\circ}$ and $\sigma=88.5^{\circ}$ lattices.
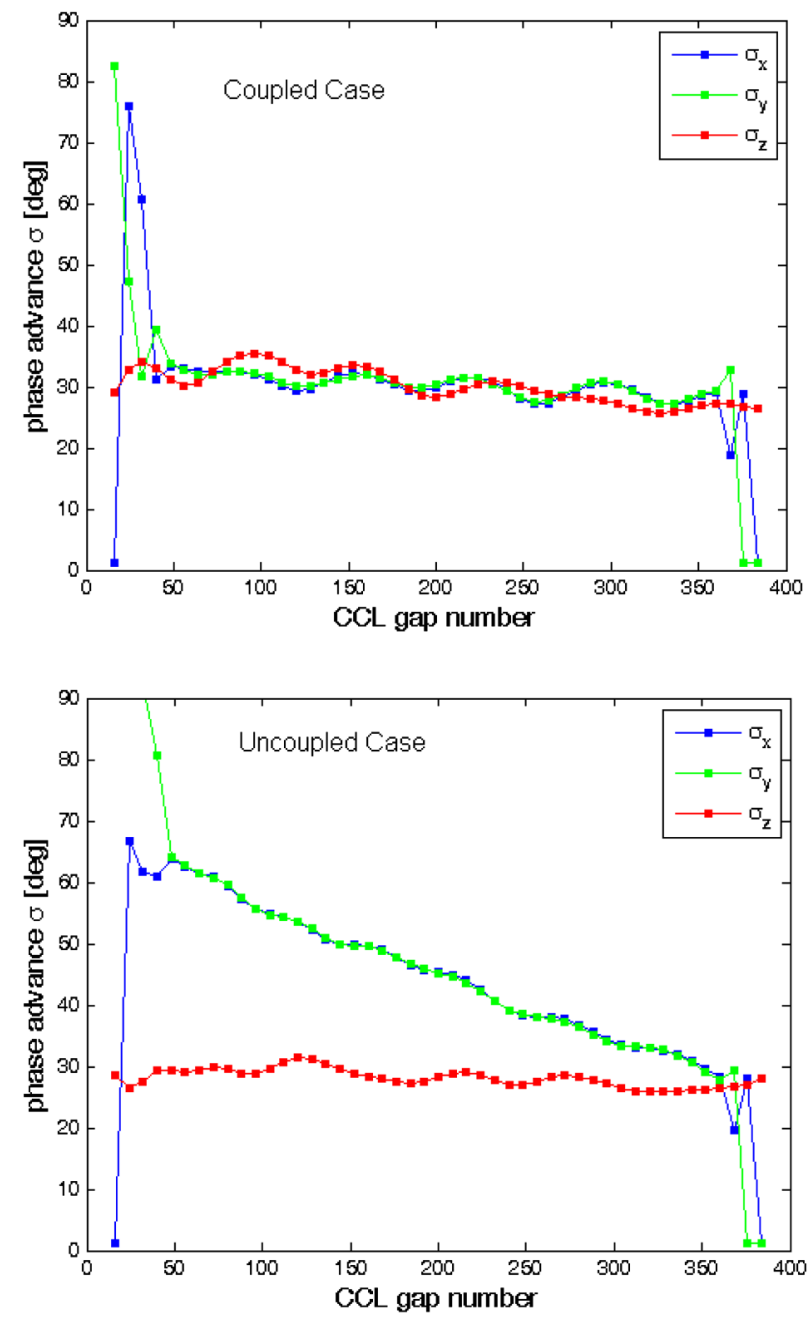

FIG. 10. Plots of transverse and longitudinal depressed phase advances per cell $\sigma_{x}, \sigma_{y}$, and $\sigma_{z}$ vs the CCL rf gap number. The top plot shows the depressed phase advances for the "coupled case" CCL lattice and the bottom plot shows the phase advances for the "uncoupled case" CCL lattice for the 38-mA beam. For the "coupled case," the transverse and longitudinal phase advances overlap with each other over the entire CCL to maximize the coupling effect. The quadrupole gradients for the "uncoupled case" is chosen such that the beam sizes of the two cases are practically equal at the end of the CCL for a zero current beam. The undepressed phase advance $\sigma_{o}$ is about $20^{\circ}$ higher than the depressed phase advance $\sigma$.
The numerical simulation indicates that the spatial extent of beam halo decreases when $\sigma$ gets close to $90^{\circ}$ (such as the case of $\sigma=88.5^{\circ}$ ) and there is no resonance effect for $\sigma>90^{\circ}$, as reported in $[16,17]$. This is due to the fact that the resonance islands shrink as $\sigma$ approaches $90^{\circ}$ and they disappear for $\sigma>90^{\circ}$. The comparison of the beam profiles for the $\sigma=65^{\circ}$ and $\sigma=88.5^{\circ}$ lattices is informative in this regard. Figure 9 shows that the beam profiles of the $\sigma=$ $88.5^{\circ}$ lattice practically overlaps with those of $\sigma=65^{\circ}$ lattice except the small beam shoulders generated by the beam particles populating the resonance islands of the $\sigma=$ $88.5^{\circ}$ lattice. This clearly demonstrates in the experiment the consistency between the experiment and the simulation of the fourth order resonance. All these agree well with the characteristics of the $4 \sigma=360^{\circ}$ space charge resonance.
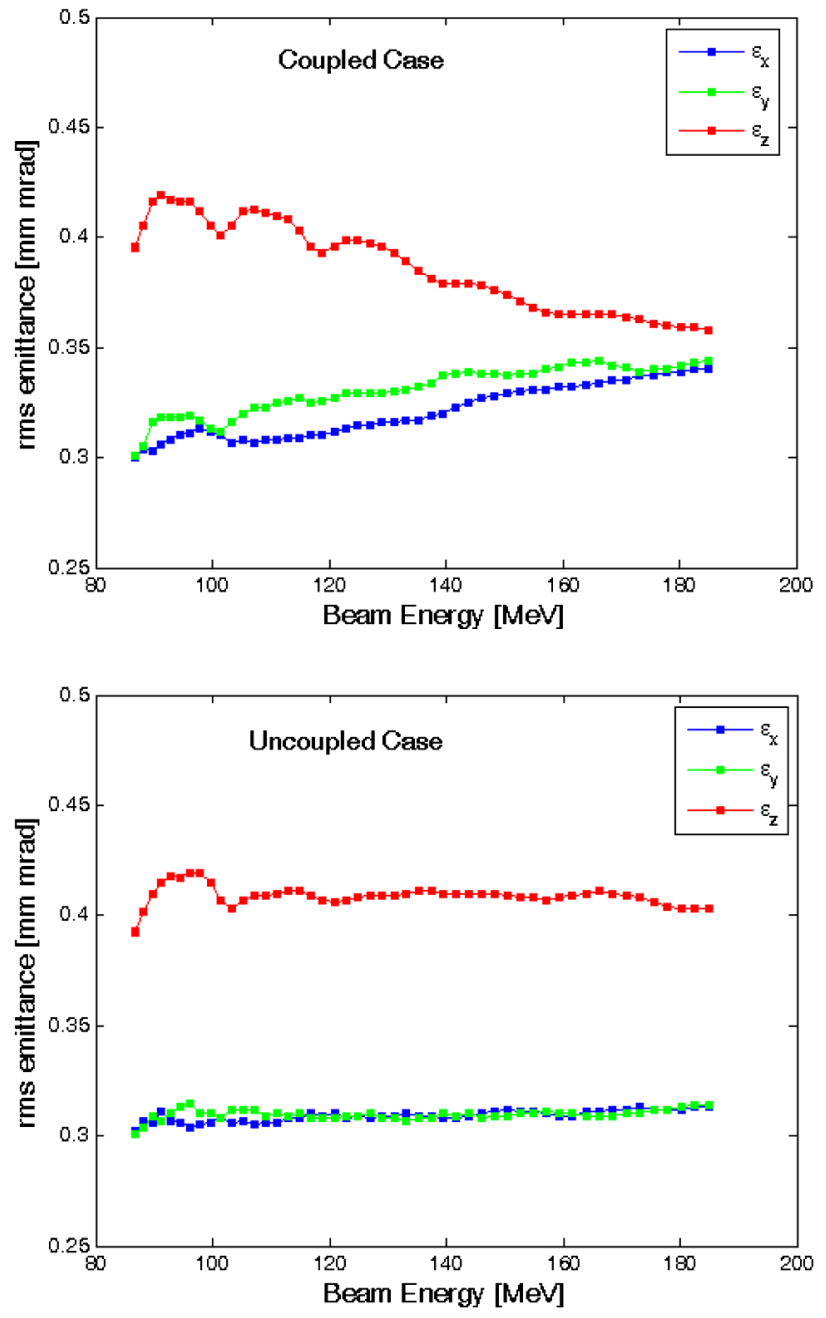

FIG. 11. Plots of transverse and longitudinal normalized rms emittances vs beam energy obtained from multi-particle simulations in the CCL. The top plot shows the rms emittances for the "coupled case" CCL lattice and the bottom plot those for the "uncoupled case" CCL lattice. For the "coupled case", one observes an emittance transfer from the longitudinal plane to the transverse planes due to the space charge coupling resonance. For the uncoupled case, there is practically no emittance transfer. 
The measurement for $\sigma>90^{\circ}$ is not done due to the interlock of the quadrupole power supplies, because the required current surpassed the interlocked values.

\section{SPACE CHARGE DRIVEN $2 \sigma_{x(y)}-2 \sigma_{z}=0$ COUPLING RESONANCE}

\section{A. Simulations of space charge driven $2 \sigma_{x(y)}-2 \sigma_{z}=0$ coupling resonance}

Simulation studies suggest the feasibility of measuring the effects of the space charge $2 \sigma_{x(y)}-2 \sigma_{z}=0$ coupling resonance between the transverse and longitudinal planes
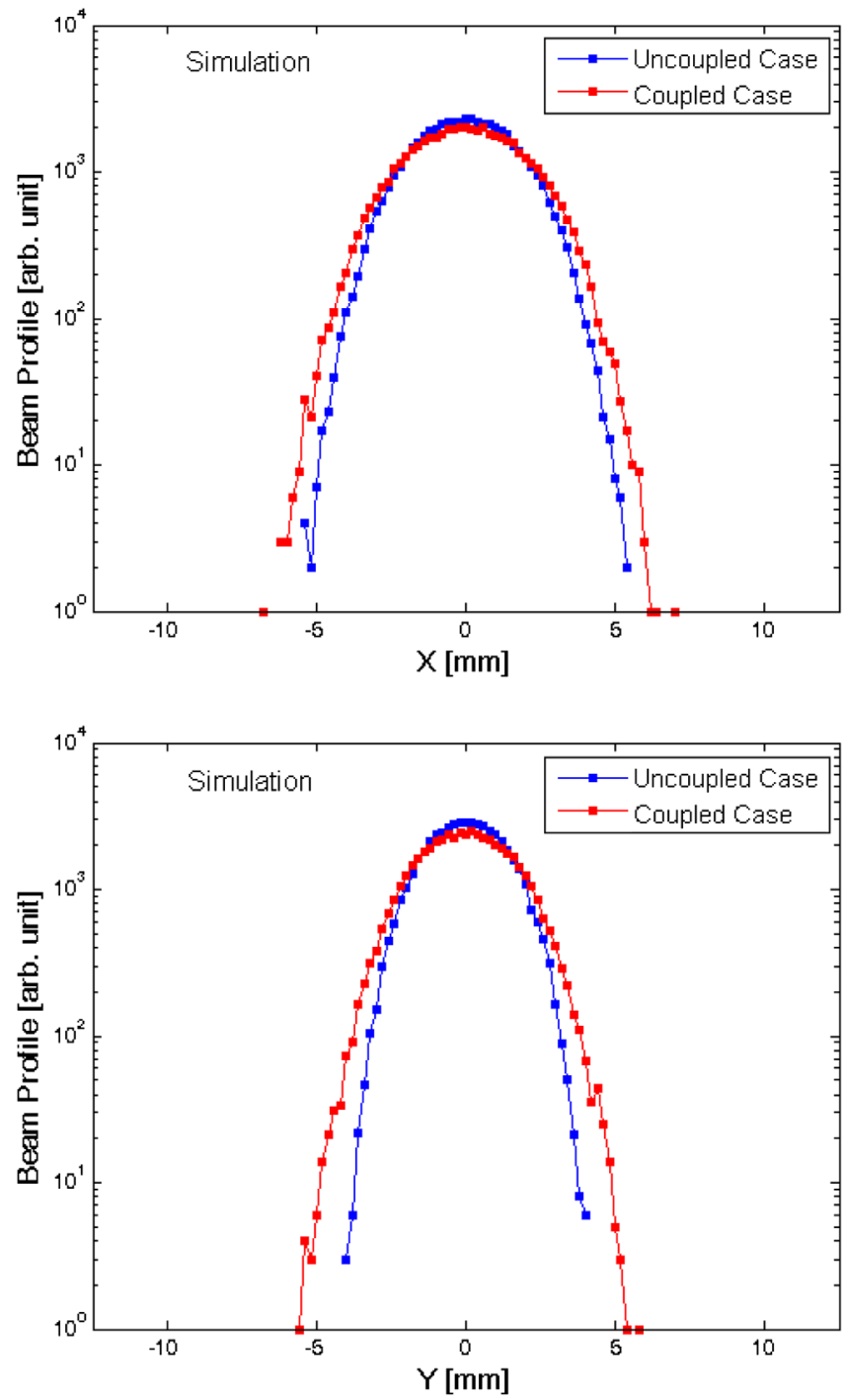

FIG. 12. Plots of the simulated beam profiles for the "coupled case" (in red) and the "uncoupled case" (in blue). The top (bottom) plot shows the simulated $\mathrm{X}(\mathrm{Y})$ beam profiles for the two cases at the location of the wire-scanner "WS310". One can notice that the space charge coupling resonance (in red) broadens beam profiles in both planes as a whole with little beam profile deformation. These are unique properties of the coupling resonance. for the 38-mA beam in the CCL. Gradients of quadrupoles are adjusted to make the depressed transverse phase advances per cell $\sigma_{x}$ and $\sigma_{y}$ overlap with the longitudinal phase advance per cell $\sigma_{z}$ to maximize the coupling resonance effect. Figure 10 shows the graphs of $\sigma_{x}, \sigma_{y}$ and $\sigma_{z}$ for the "coupled case" (the top plot) and those for the "uncoupled case" (the bottom plot) in the CCL. The uncoupled case is the design optics of the CCL. However there is a coupling at the end of the CCL (see the bottom plot of Fig. 10) and the transverse beam sizes of the two cases (coupled and uncoupled) are equal at the end of the CCL due to the same focusing strength.
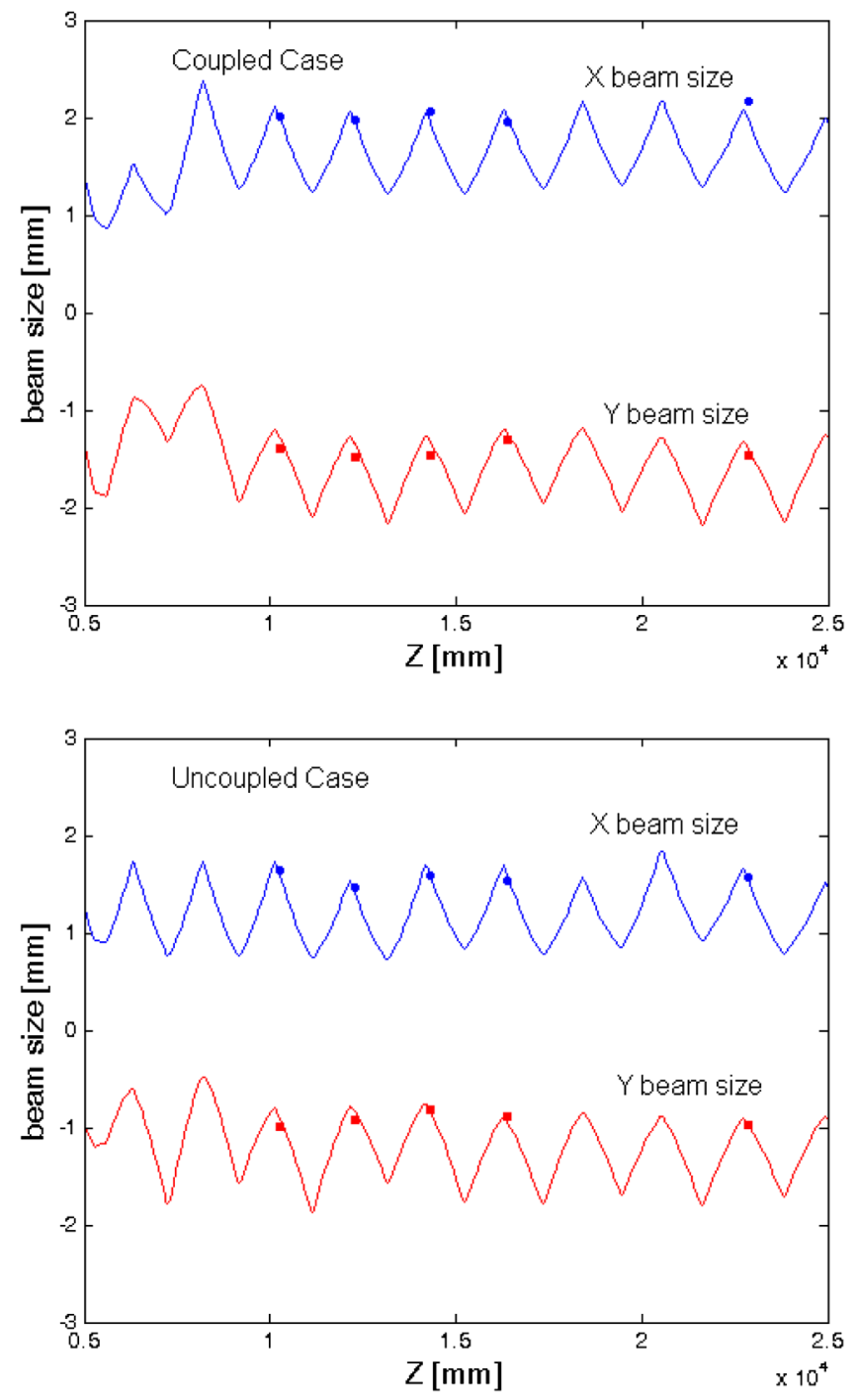

FIG. 13. Plots of the experimental beam matching into the CCL for the "coupled case" (the top plot) and for the "uncoupled case" (the bottom plot). The beam is going from the left to the right. Blue (red) solid dots represent the measured X (Y) beam sizes obtained from five wire-scanners (WS104, 106, 108, 110, 204). Solid lines are the TRACE3D code fit to the measured beam size data, which determines the incoming beam parameters $(\varepsilon, \beta, \alpha)$. Plots show that the beam is matched well into the CCL for both cases. The beam current is $38 \mathrm{~mA}$. 
For multiparticle simulations, the same 50000 macro-particle Gaussian beam distribution is used with normalized rms emittance $\varepsilon_{x}=\varepsilon_{y}=0.301 \mathrm{~mm} \mathrm{mrad}, \varepsilon_{z}=$ $0.394 \mathrm{~mm}$ mrad. Multiparticle simulations with the PARMILA code show that the emittance transfer occurs in the CCL from the longitudinal plane to the transverse planes for the "coupled case," as shown in Fig. 11. On the other hand, there is no emittance transfer for the "uncoupled case."

The space charge $2 \sigma_{x(y)}-2 \sigma_{z}=0$ coupling resonance transfers the emittance from the longitudinal plane to the transverse planes and the transverse beam profiles broaden as a whole including the core. In the CCL this resonance does not induce a beam halo formation (or shoulders) unlike the $4 \sigma=360^{\circ}$ resonance or a mismatch. Figure 12 shows the beam profile plots obtained from a multi-particle simulation with the 38-mA beam at the location of the wirescanner "WS310" for the coupled and uncoupled cases. One can note that the beam profiles broaden as a whole by
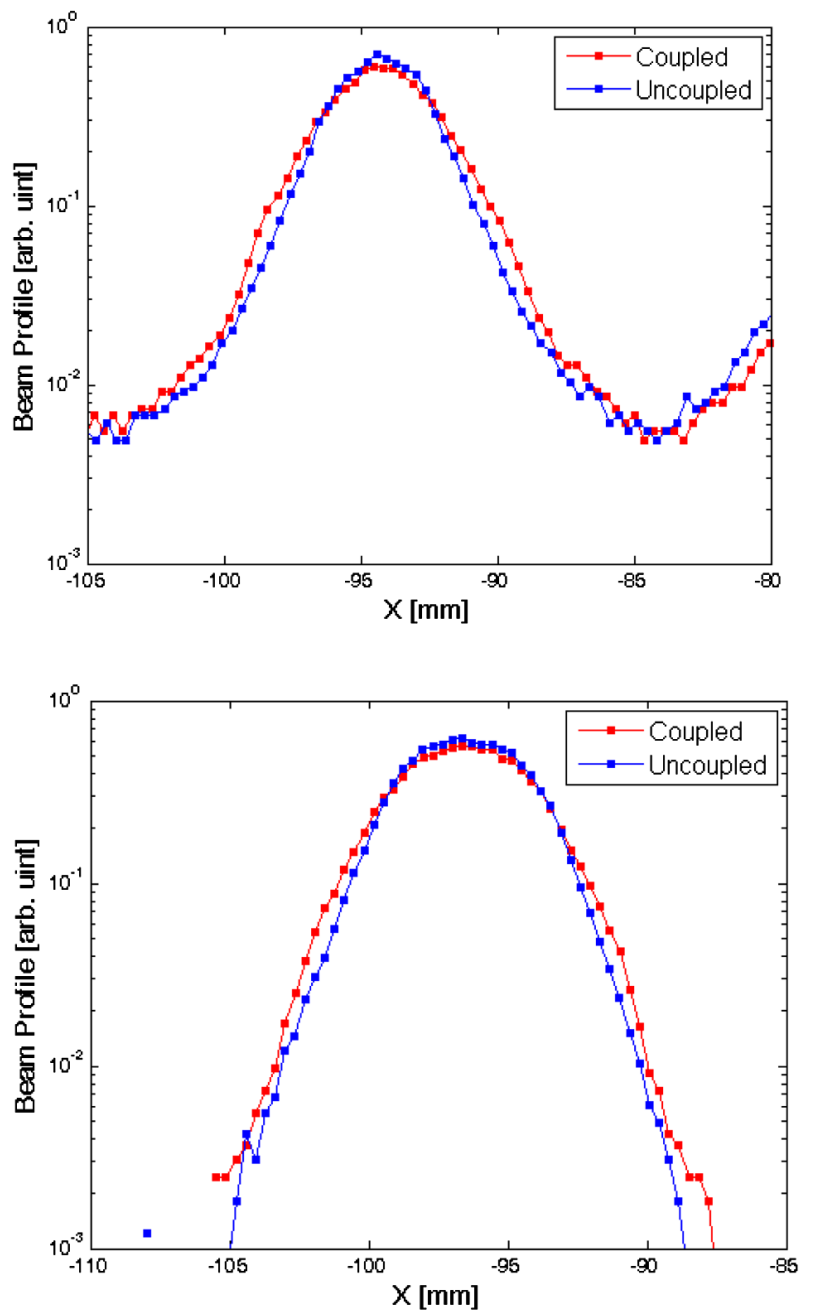

10\%-20\% in both $\mathrm{X}$ and $\mathrm{Y}$ planes due to the emittance increase through the space charge coupling resonance.

\section{B. Beam matching and incoming beam quality}

In the experiment, the beam matching is very crucial in order to minimize the possibility of beam mismatch and its halo formation. The beam is matched carefully into the CCL using the four wire-scanners in the upstream CCL, following the same methods in [22]. Figure 13 shows the results of the experimental beam matching to the CCL for the "coupled case" (the top plot) and for the "uncoupled case" (the bottom plot) for the 38-mA beam. The blue (red) solid dots represent the measured $\mathrm{X}(\mathrm{Y})$ beam sizes obtained from the five upstream wire-scanners (WS104, 106, 108, 110, 204). Solid lines are the TRACE3D code [23] fit to the measured beam size data, which determines the incoming beam parameters $(\varepsilon, \beta, \alpha)$. The quality of the
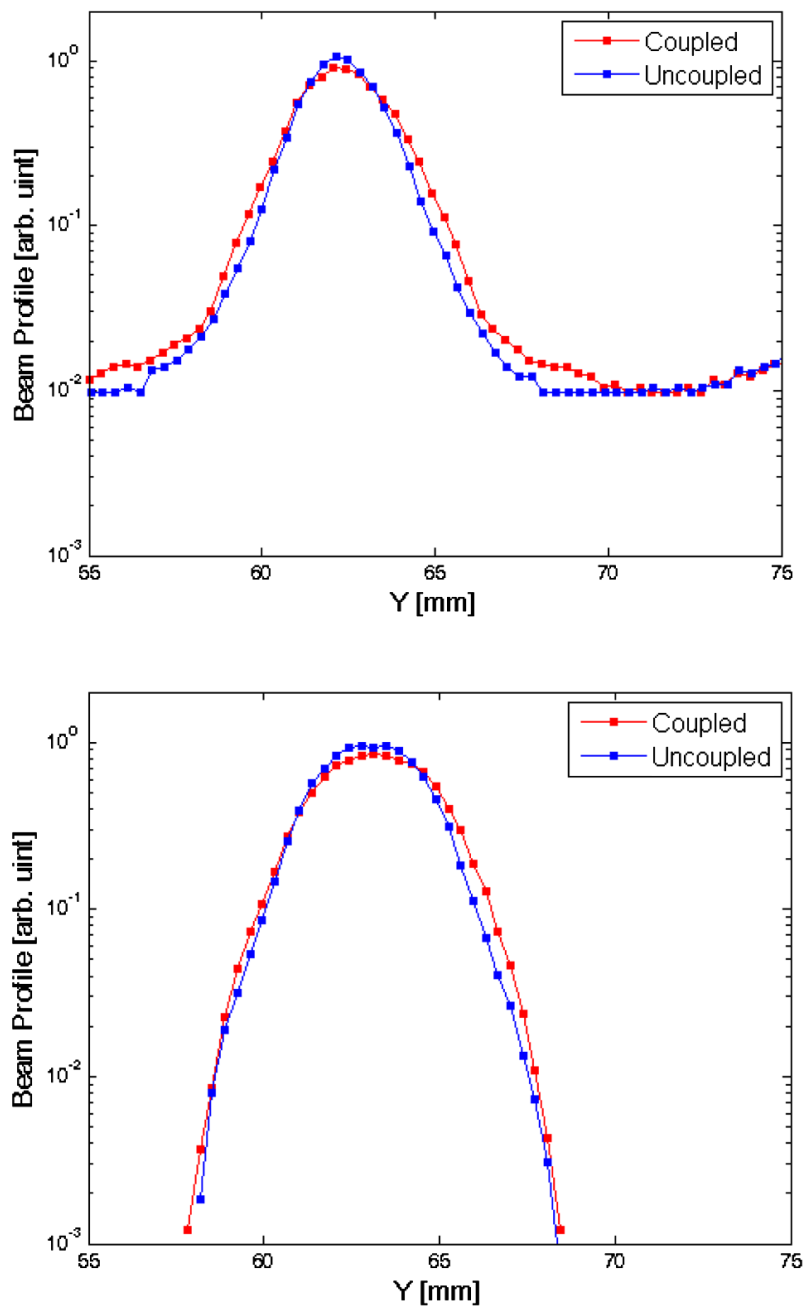

FIG. 14. Plots of the measured beam profiles of the 38-mA beam in log scale for the "coupled case" (in red) and the "uncoupled case" (in blue). The top left (right) plot shows the X (Y) beam profiles for the two cases obtained from the wire-scanner "WS310". The bottom left (right) plot shows the X (Y) beam profiles from the wire-scanner "WS406". Measured data show that the space charge coupling resonance broadens the $\mathrm{X}$ and $\mathrm{Y}$ beam profiles with little beam profile deformation. These results agree well with the simulation shown in Fig. 12. It should be noted that the measured beam profiles have little beam shoulders, indicating that there is little halo formation. 
beam going into the CCL is the same as shown in Fig. 3, because the experiment is done during the same beam study shift. The beam has very small tails and has a pretty Gaussian-like density profile.

\section{Experiment of space charge driven $2 \sigma_{x(y)}-2 \sigma_{z}=0$ coupling resonance}

Once the beam is matched into the CCL in the experiment, beam profiles are measured using the wire-scanners in the CCL for the comparison of the "coupled case" and the "uncoupled case". The two downstream wire-scanners "WS310" and "WS406" are used for the measurement, because the transverse emittances increase is maximal there
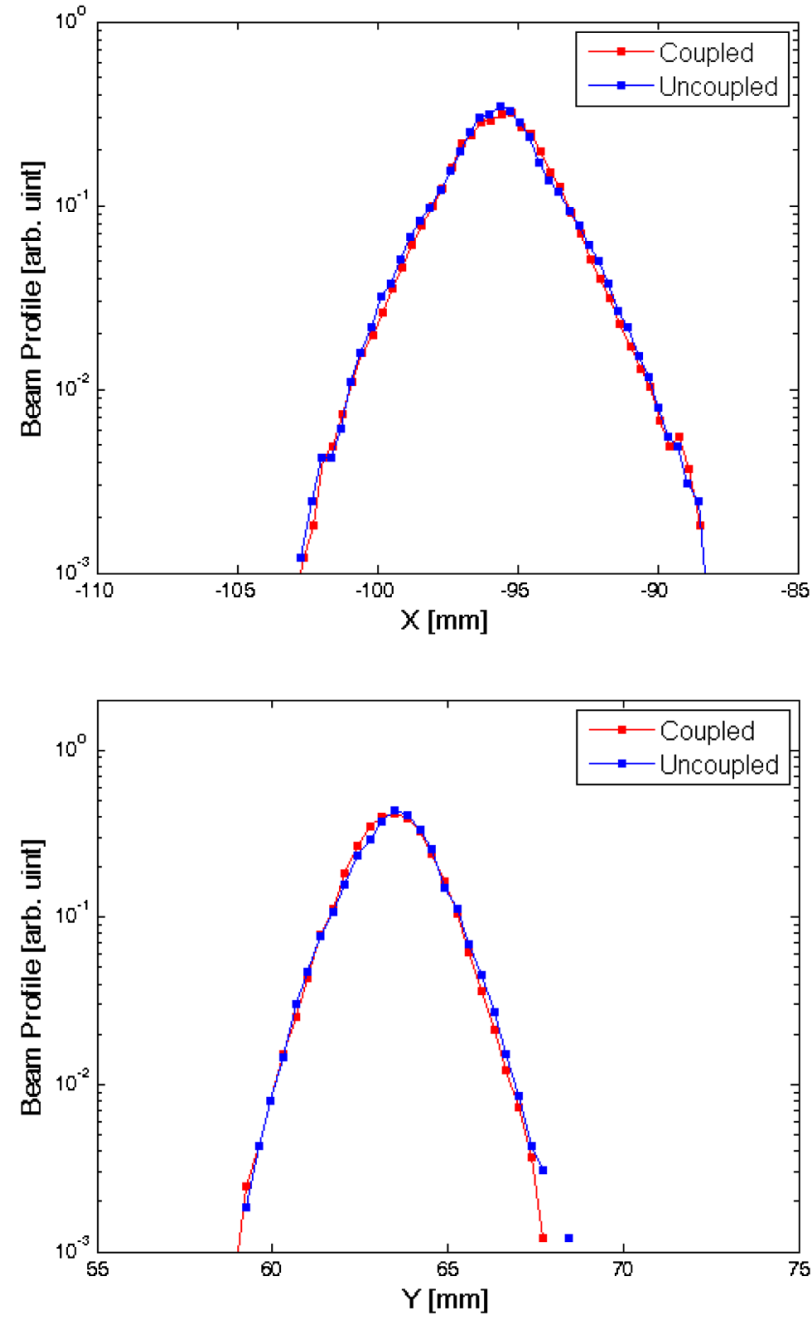

FIG. 15. Plots of the measured beam profiles of the "coupled case" (in red) and those of the "uncoupled case" (in blue) obtained from the wire-scanner WS406 for the 15-mA beam. Compared with the $38-\mathrm{mA}$ beam, the coupling resonance effects diminish and there is practically no difference in the beam profiles between the "coupled case" and the "uncoupled case" in both transverse planes. It should be noted that the measured beam profiles have little beam shoulders, indicating that there is little halo formation.
TABLE I. Measured beam size for the "coupled case" and the "uncoupled case" for the 38-mA beam.

\begin{tabular}{lcccc}
\hline \hline $\begin{array}{l}\text { Measurement } \\
\text { 38-mA }\end{array}$ & $\begin{array}{c}\text { X size } \\
\text { WS310 }\end{array}$ & $\begin{array}{c}\text { Y size } \\
\text { WS310 }\end{array}$ & $\begin{array}{c}\text { X size } \\
\text { WS406 }\end{array}$ & $\begin{array}{c}\text { Y size } \\
\text { WS406 }\end{array}$ \\
\hline Coupled case & $1.98 \mathrm{~mm}$ & $1.31 \mathrm{~mm}$ & $2.46 \mathrm{~mm}$ & $1.70 \mathrm{~mm}$ \\
$\begin{array}{l}\text { Uncoupled } \\
\text { case }\end{array}$ & $1.64 \mathrm{~mm}$ & $1.05 \mathrm{~mm}$ & $2.28 \mathrm{~mm}$ & $1.54 \mathrm{~mm}$ \\
\hline \hline
\end{tabular}

(see Fig. 11) and because the beam sizes of the two cases are practically equal for a zero current beam (see Fig. 15). These two wire-scanners are ideal for detecting the effect of the space charge coupling resonance in the CCL. For 38$\mathrm{mA}$ beam, Fig. 14 shows the comparison of measured beam profiles of the "coupled case" (in red) and the "uncoupled case" (in blue) at the last two wire-scanners of the CCL. The top left (right) plot shows the $\mathrm{X}(\mathrm{Y})$ beam profiles for the two cases obtained from the wire-scanner "WS310". The bottom left (right) plot shows the $\mathrm{X}(\mathrm{Y})$ beam profiles obtained from the wire-scanner "WS406". It should be noted that the measured $\mathrm{X}$ and $\mathrm{Y}$ beam profiles of the "coupled case" broaden as a whole with little beam profile deformation compared with the "uncoupled case." It should be noted that the measured beam profiles have little beam shoulders, indicating that there is little halo formation. This is in a good agreement with the simulation shown in Fig. 12.

These unique properties of the coupling resonance are predicted in the simulation and verified in the experiment. For the space charge coupling resonance effects in the $\mathrm{CCL}$, one does not observe the growth of beam shoulders unlike the $4 \sigma=360^{\circ}$ space charge resonance or the beam mismatch. The same measurement is repeated with a reduced beam current of $15-\mathrm{mA}$ to check the dependence of the resonance on the beam intensity. For a $15-\mathrm{mA}$ beam, there is practically no difference in the measured beam profiles between the coupled and uncoupled cases, as shown in Fig. 15. This confirms that the observed beam profile broadening for $38-\mathrm{mA}$ beam is due to the space charge induced coupling resonance.

As listed in Table I, the measured beam size of the "coupled case" at WS310 (WS406) is 20\% ( 10\%) larger than that of the "uncoupled case" for the 38-mA beam. This is consistent with the results of multiparticle simulations which show that the beam size of the "coupled case" at

TABLE II. Simulated beam size for the "coupled case" and the "uncoupled case" for the 38-mA beam.

\begin{tabular}{lcccc}
\hline \hline $\begin{array}{l}\text { Simulation } \\
38-\mathrm{mA}\end{array}$ & $\begin{array}{c}\mathrm{X} \text { size } \\
\text { WS310 }\end{array}$ & $\begin{array}{c}\text { Y size } \\
\text { WS310 }\end{array}$ & $\begin{array}{c}\text { X size } \\
\text { WS406 }\end{array}$ & $\begin{array}{c}\text { Y size } \\
\text { WS406 }\end{array}$ \\
\hline Coupled case & $2.09 \mathrm{~mm}$ & $1.38 \mathrm{~mm}$ & $2.13 \mathrm{~mm}$ & $1.34 \mathrm{~mm}$ \\
$\begin{array}{l}\text { Uncoupled } \\
\text { case }\end{array}$ & $1.83 \mathrm{~mm}$ & $1.13 \mathrm{~mm}$ & $1.94 \mathrm{~mm}$ & $1.25 \mathrm{~mm}$ \\
\hline \hline
\end{tabular}


TABLE III. Simulated beam size for the "coupled case" and the "uncoupled case" for the 0 -mA beam.

\begin{tabular}{lcccc}
\hline \hline Simulation & X size & Y size & X size & Y size \\
0-mA & WS310 & WS310 & WS406 & WS406 \\
\hline Coupled case & $1.63 \mathrm{~mm}$ & $1.02 \mathrm{~mm}$ & $1.62 \mathrm{~mm}$ & $1.04 \mathrm{~mm}$ \\
Uncoupled & $1.61 \mathrm{~mm}$ & $0.94 \mathrm{~mm}$ & $1.62 \mathrm{~mm}$ & $1.06 \mathrm{~mm}$ \\
$\quad$ case & & & & \\
\hline \hline
\end{tabular}

WS310 (WS406) is 20\% ( 10\%) larger than that of the "uncoupled case" for the 38-mA beam, as listed in Table II. On the other hand, for a zero current beam (with the same beam emittance as the $38-\mathrm{mA}$ beam), the multiparticle simulations show that the beam sizes of the two cases become practically equal, as listed in Table III.

\section{SUMMARY}

Experimental evidences for the space charge $4 \sigma=360^{\circ}$ resonance and the space charge $2 \sigma_{x(y)}-2 \sigma_{z}=0$ coupling resonance of high intensity linear accelerators are presented that are obtained from the SNS CCL. The experiment is conducted with an excellent control of beam quality minimizing the beam halo and beam mismatch into the CCL. Beam profile measurements verify that the beam going into the CCL has indeed very little beam tails and that the beam is matched well into the CCL, which excludes the possibility of mismatch of the beam going into the CCL.

The effects of the $4 \sigma=360^{\circ}$ space charge resonance are measured with multiple wire-scanners. A good agreement is obtained between the experiment and simulation. The experiment confirms unique characteristics of the space charge $4 \sigma=360^{\circ}$ resonance that the simulation shows, especially as the phase advance $\sigma$ approaches $90^{\circ}$. These characteristics are related to the fact that the stable islands shrink and move toward the origin as the phase advance per cell $\sigma$ approaches $90^{\circ}$. The experimental results clearly show that the resonance effect diminishes as $\sigma$ gets very close to $90^{\circ}$.

The $2 \sigma_{x(y)}-2 \sigma_{z}=0$ space charge coupling resonance effects are measured using multiple wire-scanners. As the simulation predicts, the experiment verifies that the space charge coupling resonance in the CCL induces 10-20\% beam size increase in both $\mathrm{X}$ and $\mathrm{Y}$ planes at the last two CCL wire-scanners. It is also verified that the beam profile broadens as a whole unlike the mismatch or the fourth order resonance. In case of the mismatch or the fourth order resonance, beam halo is generated and the beam tail profiles are altered. Multiparticle simulations with a zero current beam show that the beam size of the coupled and uncoupled cases become practically equal. The experiment with the $15-\mathrm{mA}$ beam shows that the beam size of the two cases become practically equal, and confirms that the observed effects are due to the space charge coupling resonance.

\section{ACKNOWLEDGMENTS}

This work was supported in part by the Rare Isotope Science Project of the Institute for Basic Science funded by the Ministry of Science, ICT and Future Planning (MSIP) and the National Research Foundation (NRF) of the Republic of Korea under Contract No. 2013M7A1A1075764. This work was also supported in part by the SNS managed by UT-Battelle, LLC, under Contract No. DE-AC05-00OR22725 for the U.S. Department of Energy.

[1] J. Stovall et al., in Proceedings of the Particle Accelerator Conference, Chicago, IL, 2001 (IEEE, New York, 2001), p. 446.

[2] Y. Yamazaki, in Proceedings of the 2003 Particle Accelerator Conference, Portland, OR (IEEE, New York, 2003), p. 576.

[3] B.-H. Choi, Proc. of 2004 Asian Part. Accl. Conf., Gyeongju, Korea (IEEE, New York, 2004), p. 231.

[4] H.F. Ouyang et al., in Proceedings of the 25th International Linear Accelerator Conference, LINAC2010, Tsukuba, Japan (KEK, Tsukuba, Japan, 2010), p. 362.

[5] D. Jeon et al., J. Korean Phys. Soc. 65, 1010 (2014).

[6] J. Wei et al., Proc. of 2012 Linear Accl. Conf., Tel-Aviv, Israel (IEEE, New York, 2012), p. 417.

[7] F. J. Sacherer, IEEE Trans. Nucl. Sci. 18, 1105 (1971); P. M. Lapostolle, IEEE Trans. Nucl. Sci. 18, 1101 (1971).

[8] J. S. O'Connell, T. P. Wangler, R. S. Mills, and K. R. Crandall, in Proceedings of the 15th Particle Accelerator Conference, PAC-1993, Washington, DC, 1993 (IEEE, New York, 1993), p. 3657.

[9] J.-M. Lagniel, Nucl. Instrum. Methods Phys. Res., Sect. A 345, 46 (1994); R. L. Gluckstern, Phys. Rev. Lett. 73, 1247 (1994).

[10] J.-M. Lagniel and D. Libault, in Proceedings of the Particle Accelerator Conference, Dallas, TX, 1995 (IEEE, New York, 1995), p. 446.

[11] T. P. Wangler, K. R. Crandall, R. Ryne, and T. S. Wang, Phys. Rev. ST Accel. Beams 1, 084201 (1998).

[12] I. Hofmann, L. J. Laslett, L. Smith, and I. Harber, Part. Accel. 13, 145 (1983).

[13] J. Struckmeier and M. Reiser, Part. Accel. 14, 227 (1984).

[14] D. Jeon, J. A. Holmes, V. V. Danilov, J. D. Galambos, and D. K. Olsen, Phys. Rev. E 60, 7479 (1999).

[15] G. Franchetti, I. Hofmann, and D. Jeon, Phys. Rev. Lett. 88, 254802 (2002).

[16] D. Jeon, L. Groening, and G. Franchetti, Phys. Rev. ST Accel. Beams 12, 054204 (2009).

[17] L. Groening, W. Barth, W. Bayer, G. Clemente, L. Dahl, P. Forck, P. Gerhard, I. Hofmann, M. S. Kaiser, M. Maier, S. Mickat, T. Milosic, D. Jeon, and D. Uriot, Phys. Rev. Lett. 102, 234801 (2009).

[18] D.-O. Jeon, K. R. Hwang, J.-H. Jang, H. Jin, and H. Jang, Phys. Rev. Lett. 114, 184802 (2015).

[19] M. Reiser, Theory and Design of Charged Particle Beams (John Wiley \& Sons Inc., New York, 1994), p. 240. 
[20] C. Li and Y.L. Zhao, Phys. Rev. ST Accel. Beams 17, 124202 (2014).

[21] H. Takeda and J. Stovall, in Proceedings of the Particle Accelerator Conference, Dallas, TX, 1995 (IEEE, New York, 1995), p. 2364.

[22] D. Jeon, C. M. Chu, J. Stovall, and S. Assadi, Nucl. Instrum. Methods Phys. Res., Sect. A 607 (2009) 517; D. Jeon and J. Stovall, in Proceedings of the 2003 Particle Accelerator Conference, Portland, OR (IEEE, New York, 2003), p. 2652; D. Jeon, S. Assadi, and J. Stovall, Proc. of the 2002 Linear Accl. Conf., Gyeongju, Korea (IEEE, New York, 2002), p. 88.

[23] K. Crandall and D. Rusthoi, Los Alamos National Laboratory Report No. LA-10235-MS, 1985.

[24] D. Jeon, J. Stovall, A. Aleksandrov, J. Wei, J. Staples, R. Keller, L. Young, H. Takeda, and S. Nath, Phys. Rev. ST Accel. Beams 5, 094201 (2002).

[25] D.-O. Jeon, Phys. Rev. ST Accel. Beams 16, 040103 (2013). 